\title{
Adaptive Transmit Power Control Algorithm for Sensing-Based Semi-Persistent Scheduling in C-V2X Mode 4 Communication
}

\author{
Amir Haider ${ }^{(D)}$ and Seung-Hoon Hwang * (D) \\ Division of Electronics and Electrical Engineering, Dongguk University-Seoul, Seoul 04620, Korea \\ * Correspondence: shwang@dongguk.edu; Tel.: +82-2-2260-3994
}

Received: 5 June 2019; Accepted: 25 July 2019; Published: 29 July 2019

\begin{abstract}
For cellular-based vehicle-to-everything (C-V2X) communication, vital information about status and intention is periodically broadcasted by each vehicle using the cooperative awareness message (CAM) service. In C-V2X, the task of resource allocation can either be carried out in a centralized manner by the network, termed Mode 3, or by the vehicles themselves in a distributed manner without any core network support, termed Mode 4 . Mode 4 scheduling is accomplished by employing sensing-based semi-persistent scheduling (SB-SPS), where the vehicles sense the medium and identify the best time-frequency resource combination for transmission of the CAM. Focusing on Mode 4 in this paper, we present a comprehensive analysis of the impact of variations in the transmit power of the vehicle on the performance of SB-SPS for C-V2X communications in various traffic scenarios through simulations. An adaptive-transmit power control (A-TPC) algorithm is presented to improve the quality of service for various large-scale traffic scenarios, where each vehicle uses real-time channel-sensing information to adjust the transmit power in order to avoid interference with neighbouring vehicles. The results demonstrate that our proposed algorithm outperforms the conventional TPC schemes.
\end{abstract}

Keywords: LTE; C-V2X services; C-V2V communication; 5G; SB-SPS; transmit power control

\section{Introduction}

To transform the future automotive industry, extensive research is being conducted on cellular-based vehicle-to-everything (C-V2X) communications to support various services such as autonomous driving, road safety, and accident reduction, leading to the prevention of accidents and providing more efficient traffic management [1]. To provide such services, information exchange is enabled among vehicles $(\mathrm{C}-\mathrm{V} 2 \mathrm{~V})$, infrastructure $(\mathrm{C}-\mathrm{V} 2 \mathrm{I} / \mathrm{V} 2 \mathrm{~N})$, and pedestrians $(\mathrm{C}-\mathrm{V} 2 \mathrm{P})$ via $\mathrm{C}-\mathrm{V} 2 \mathrm{X}$ communication as shown in Figure 1. C-V2X can be envisioned as an extension for dedicated short-range communication (DRSC), as both technologies deal with user equipment (UE) control signaling [2]. Proximity services for device-to-device (D2D) communication listed in the 3rd generation partnership project (3GPP) Release 12 enable UEs to exchange data over short distances by establishing a direct link between the devices. Using this approach, long term evolution (LTE) traffic at the eNodeB can be efficiently offloaded [3]. Additionally, the UEs can relay data to neighbouring devices with poor connectivity, thus extending the coverage range for the network [4]. For such short-range D2D communications, two modes, namely Modes 1 and 2, have been defined by the 3GPP specifications. While Mode 1 operates under network-controlled scheduling, Mode 2 employs autonomous scheduling without the support of the core network. The sole purpose of these two modes is to improve the UE battery life along with the network range extension mentioned earlier [5]. 


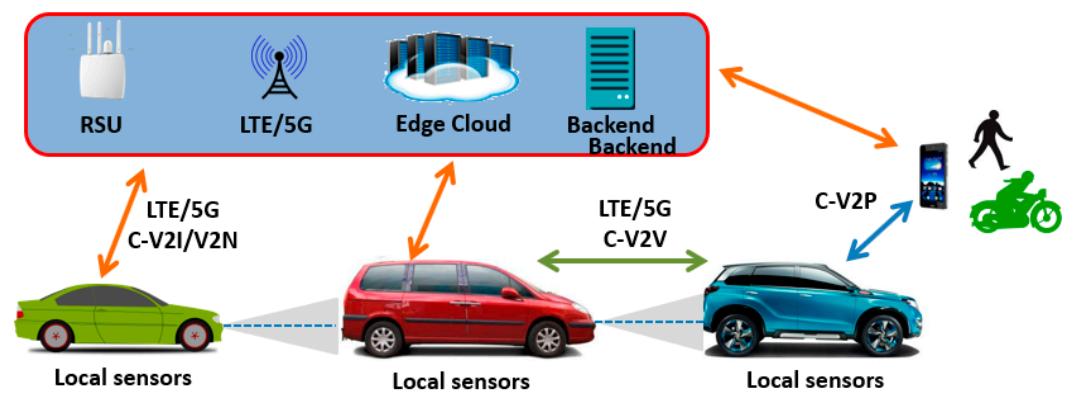

Figure 1. Envisioned cellular-based vehicle-to-everything (C-V2X) communication system.

In recent years, vehicular ad hoc networks (VANETs) have gained a lot of popularity in implementation of various applications in V2X communications, particularly for road safety and efficient traffic management. The vehicles form an ad hoc network by using the on-board units (OBUs) that allow wireless communication for various V2X use cases [6]. In terms of practical implementation, one of the key protocols adopted for VANETs is the wireless access in vehicular environment (WAVE). WAVE protocol utilizes the IEEE 802.11p standard and provides basic radio standard for dedicated short-range communication (DSRC) operations in $5.9 \mathrm{GHz}$ frequency band [7]. Several recent works in literature demonstrated the real-time implementation of the VANETs. A detailed survey on the wireless access standards, technological status, and future challenges, and various trials and simulators for VANETs was presented in [8]. Considering the safety and traffic congestion avoidance, authors in [9] proposed a secure communication system for self-organized vehicular networks, termed as VAiPho algorithms along with the software which employs the Wi-Fi and GPS equipped smart phones only. However, compared to LTE and other future wireless technologies, the proposed system cannot provide higher transmission speed, longer range of transmission, and large network throughput. By considering the challenges in real environment while implementing VANETs, authors in [10] addressed the issues of IP duplication caused due to existence of sub-networks on different channels as well as the devices with the same IP address on same channel. This induces interference between the wireless channels and thus results in performance degradation and can be avoided by adopting a fuzzy logic approach of merging the smaller VANETs to the large ones. The approach was validated by extensive simulations and implemented using real devices. However, the merging of sub-networks takes longer time as the sub-net size increases, thus inducing latency in communication, which is not favourable in various V2X applications. To improve the reliability in D2D communications, a reliable multicast approach for D2D communications was described in [11] along with the integration of cellular network. The proposed approach enabled direct as well as cellular mode communication among the devices by classifying closely located devices into clusters, hence providing an efficient solution to meet the high data traffic requirements for dense networks. However, the impact of variation in transmission power was not considered, which can have considerable impact on the system performance. To reduce the number of packet collisions due to high density of traffic, an efficient clustering mechanism to reduce the number of transmissions in VANETs was proposed in [12]. Although such mechanism can significantly decrease the data overhead, the issue of information gap remains unaddressed, where the vital information for various V2X use cases is limited due to insufficient transmission by the vehicles.

Similar to the legacy VANETs and D2D communications, C-V2X communication is constrained by various factors, such as the need for low latency and the accommodation of high mobility and high user density [13-15]. To accommodate a seamless and reliable exchange of information among vehicles, roadside units, and vulnerable pedestrians in the current LTE and future 5G networks, 3GPP introduced the C-V2X communication features in Release 14 [16,17]. Two additional modes of operation termed Mode 3 and Mode 4 were introduced based on the scheduling preferences illustrated in Figure 2. Mode 3 employs a centralized scheduling approach under the coverage of LTE eNodeB, where two vehicles can communicate directly, but the selection of the sub-channels or radio resources for $\mathrm{C}-\mathrm{V} 2 \mathrm{~V}$ transmission are managed by the control signaling from the cellular infrastructure over the 
Uu interface (uplink and downlink). Mode 4 adopts the new PC5 interface for direct communication among vehicles without the need for coverage from the LTE eNodeB. In summary, C-V2X extends the D2D communication specification by employing two additional modes of operation focused on V2V communication for vehicles within and outside the coverage range of the existing LTE infrastructure. For the C-V2X communication situation under consideration, Mode 3 scheduling is limited by the following major factors in comparison to Mode 4 in which vehicles select their transmission resources in an autonomous manner:

- $\quad$ To be scheduled via Mode 3, the vehicles must stay connected to the LTE network. This imparts a significant burden on mobility management, especially for scenarios where vehicles have high mobility, such as highway scenarios.

- In the event of lost coverage, Mode 3 can no longer perform resource allocation, thus completely blocking V2X transmissions.

Therefore, Mode 4 is undoubtedly considered the baseline mode for C-V2X communication.
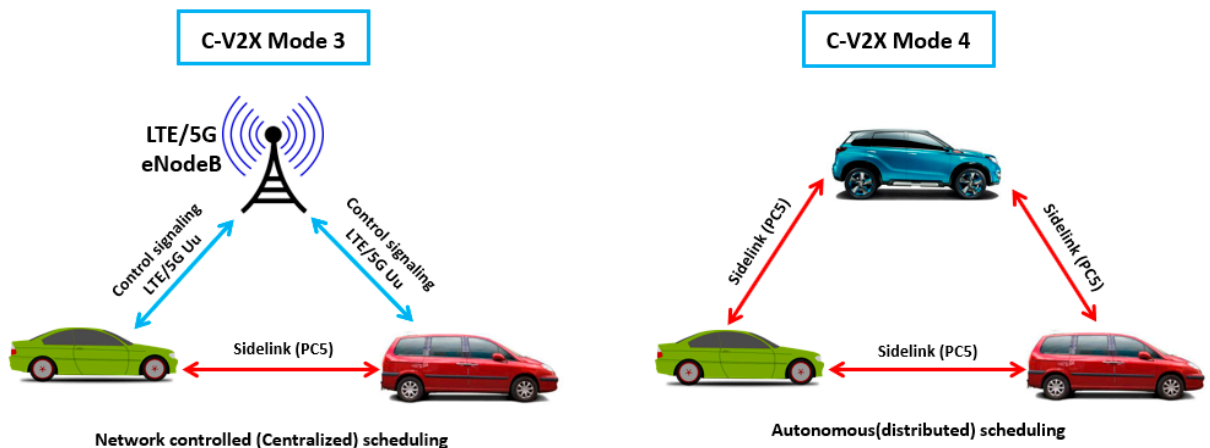

Figure 2. Illustration of scheduling modes for C-V2X communication system.

For C-V2X communication, vital information about the status and intention is periodically broadcasted by each vehicle using cooperative awareness messages (CAMs). The standard CAM is one of the component of the reference architecture defined by the European Telecommunication Standards Institute (ETSI) specifications for Intelligent Transport Systems (ITS) in [18]. CAM is the ability of the vehicle to periodically broadcast basic status information to neighbouring vehicles. With the information provided by vehicles using CAMs, vehicles can create a virtual map of their surrounding conditions to be used for several C-V2X use cases defined in [19], such as adaptive cruise control, autonomous braking, platooning, and autonomous driving. Figure 3 depicts the proposed use cases for C-V2X. To deal with CAMs for C-V2X communication using the Mode 4 algorithm, sensing-based semi-persistent scheduling (SB-SPS) is adopted in this work. Concerning the transmit power control (TPC) mechanism, C-V2X communication has a fixed maximum transmit power of $23 \mathrm{dBm}$ defined by 3GPP [16]. In addition, ETSI requirements for ITS stations [18], aimed at reducing interference from vehicles on the tolling system for DSRC, recommend that upon the detection of a tolling system, vehicles must reduce the transmit power to $10 \mathrm{dBm}$ over the sidelink. Taking the ETSI ITS recommendations into account, the Mode 4 algorithm at 10dbm transmit power can be adopted to reduce the interference among the vehicles as well. Unlike legacy LTE uplinks, where the UE can adjust the transmit power based on the TPC command from the eNodeB [20], C-V2X utilizes a fixed transmit power level without any TPC mechanism to avoid interference. Therefore, it is critical to carefully analyse the power control procedure and there is a need for an efficient power control algorithm. Taking this into account, in this work we propose an adaptive-transmit power control (A-TPC) mechanism that operates during the reallocation of CAM resources in the 3GPP Mode 4 algorithm. The proposed algorithm is described in detail in Section 3. 

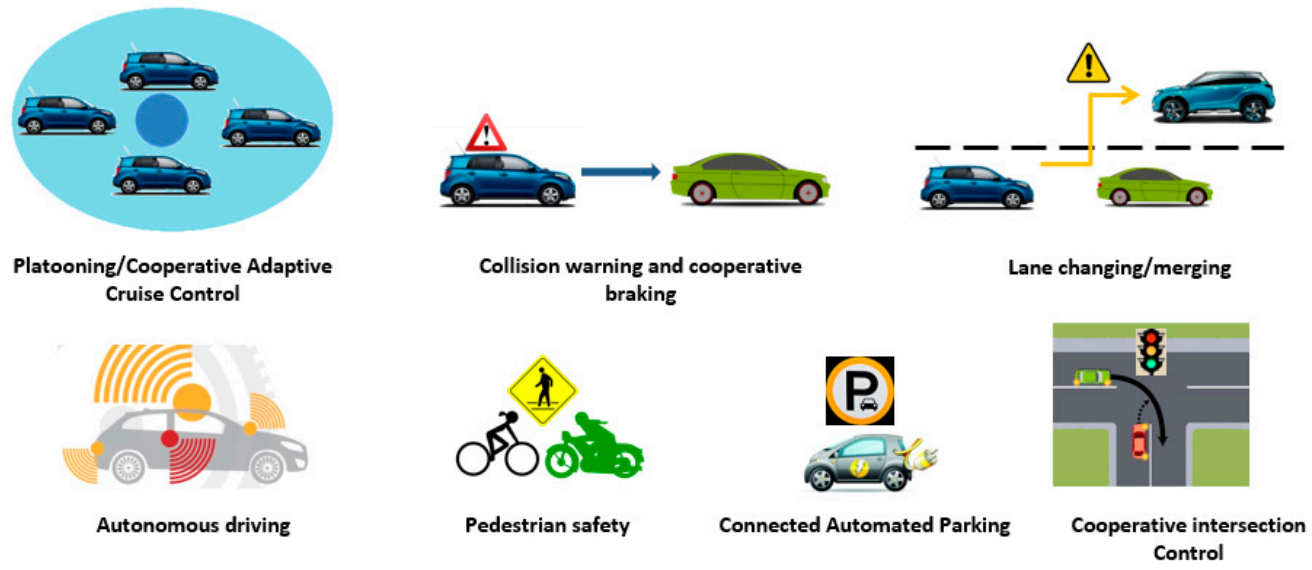

Figure 3. Proposed use cases facilitated by C-V2X support.

In the literature, several recent papers focused on the Mode 4 algorithm by adopting various resource allocation schemes. In [21], the authors presented a system-level evaluation for LTE-V2V to demonstrate the distributed scheduling of the Mode 4 algorithm in an urban scenario. The goal was to identify various transmission errors that occur while employing the SB-SPS protocol. In particular, the hidden-node problem that occurs with increasing distance between the transmitter and receiver was identified in $\mathrm{V} 2 \mathrm{~V}$ communications. An adaptive transmission scheme to adjust the modulation level by targeting the data rates of vehicles was presented in [22]. The proposed scheme considered the number of neighbouring vehicles to adjust the modulation level, and the results suggested that the modulation level must be adjusted for variations in the traffic density to optimize the system performance. By examining cooperative awareness in V2X communication, an extensive comparison of IEEE 802.11p and LTE-V2V was presented in [23], with a focus on determining the maximum awareness range to avoid the hidden-node problem. Similarly, an analytical model was presented in [24] to evaluate the packet delay and packet drop rate using $802.11 \mathrm{p}$ broadcasting for the platooning scenario. A comparison between the Mode 4 algorithm with 802.11p and the DSRC protocol was presented in [25], which inferred that Mode 4 with SB-SPS can be adopted for sidelink communications. However, the need to carefully investigate the SB-SPS parameters was stressed. An SB-SPS scheduling approach to reduce the collision of CAM transmissions was presented in [26] by employing reselection lookaheads in C-V2X. In [27], the authors outlined several analytical models for performance evaluation in C-V2X Mode 4 for the first time, particularly the packet delivery ratio (PDR). The proposed analytical models were extensively investigated for various transmission parameters including the transmission power, packet transmission rate, and modulation and coding schemes (MCS). Concerning the transmission power, two fixed values of 23 and $20 \mathrm{dBm}$ were employed. However, the analysis was limited to only setting the transmit power to ensure the received power level reached a certain threshold. To investigate the performance of SB-SPS, an open-source simulator implemented in ns-3 was presented in [28], along with optimization of the SB-SPS parameters. However, the transmit power was not considered. System-level simulation analysis for the physical and MAC layer parameters was presented in [29], but the role of transmit power control was not investigated. An SB-SPS power adaptation algorithm was proposed in [30], where the transmit power was set to 23 or $10 \mathrm{dBm}$ based on the traffic density. The scope of this algorithm was only limited to the highway scenario and SB-SPS parameter optimization was left for future consideration. Although several investigations on the performance enhancement of C-V2X Mode 4 were carried out recently, they all were limited to specific performance metrics, one or a few parameters, and traffic scenarios. To the best of our knowledge, the TPC mechanism for C-V2X Mode 4 has not been investigated much until now but can provide a significant performance improvement.

In this work, we propose the A-TPC algorithm with SB-SPS for C-V2X Mode 4. The main contributions of this work are as follows: 
- $\quad$ The formulation for the A-TPC algorithm with SB-SPS for C-V2X Mode 4.

- Performance comparison of the proposed algorithm with the power adaptation algorithm presented in [30].

- $\quad$ Performance evaluation of the proposed A-TPC algorithm by employing 3GPP Standard Mode 4 settings in three realistic traffic scenarios, which are:

Urban scenario with medium traffic density [31],

- Urban scenario with congested traffic [32],

- Highway scenario with high traffic density [32].

The rest of the paper is organized as follows: The 3GPP C-V2X Mode 4 algorithm is elaborated in Section 2. The proposed system model explaining the simulation setup and power control algorithm is presented in Section 3. The simulation results for the proposed algorithm are discussed in Section 4. Finally, Section 5 presents the conclusion of our work and future directions.

\section{3GPP C-V2X Mode 4 Algorithm}

For sidelink vehicular communication, C-V2X supports 10 and $20 \mathrm{MHz}$ channels on the $5.9 \mathrm{GHz}$ ITS band. At the physical and MAC layers, it utilizes single-carrier frequency-division multiple access (SC-FDMA) similar to the LTE uplink. The time-frequency domains are organized into orthogonal resources called resource blocks (RBs). The allocation of RBs is done in pairs, constituting $180 \mathrm{kHz}$ of bandwidth. In terms of frequency, each RB contains 12 subcarriers with an inter-spacing of $15 \mathrm{kHz}$. In the time domain, each RB has a duration of $1 \mathrm{~ms}$. This time duration corresponds to 14 orthogonal frequency-division multiplexing (OFDM) symbols. Of these, nine symbols are utilized to carry data, four symbols are reserved for channel estimation, and one symbol is used for time adjustment and a possible indication of transmitter-receiver switch. The transmission time interval (TTI) is defined as $1 \mathrm{~ms}$, which corresponds to the minimum allocation time. In the frequency domain, the RBs are grouped to form subchannels with a size defined by the network. The data packet to be carried by the subchannel is termed the transport block (TB). Sidelink control information (SCI), constituted by two $\mathrm{RBs}$, is the control message associated with each TB. The transmission of each TB and its associated SCI must be carried out in the same subframe. The allocation, however, can be done either on adjacent or non-adjacent RBs. SCI transmission is done in the first allocated subchannel for adjacent allocation, while for the non-adjacent case, TBs occupy the subchannels and specific resource reservation is needed for SCI. Various parameters such as the allocation type, size of the subchannel, size of the TB and the adopted MCS determine the number of subchannels allocated to carry a packet.

To maintain the traffic flow, each vehicle utilizes the cooperative awareness service to broadcast CAMs to update neighbouring vehicles about its status and other safety information. 3GPP Mode 4 employs SB-SPS to reserve subchannels for a defined time interval. The physical and MAC layer parameters for SB-SPS for C-V2X Mode 4 are shown in Table 1. The definition along with the standard values defined by 3GPP are listed as well. One of the key objectives of the Mode 4 algorithm is to determine the appropriate group of subchannels for CAM transmission to ensure error-free reception by the neighbouring vehicles. This group of subchannels will be referred to as "CAM resources" in the rest of the paper. The CAM resources are selected through channel sensing and are reserved for future use. The procedure for the SB-SPS scheme is shown in Figure 4. The steps performed by each vehicle to determine the CAM resources for transmission using SB-SPS are as follows:

- $\quad$ Step 1. Channel sensing: To sense the channel, each vehicle continuously monitors the channel by measuring the sidelink received signal strength indication (SL-RSSI) for each subchannel to determine the interference level for every subframe. These sensing measurements are collected for a defined sensing time $T_{\text {sense }}$. 
- $\quad$ Step 2. Listing available CAM resources: Following the sensing measurements, each vehicle enlists its available CAM resources, termed $L_{C A M-R}$. The selection of the CAM resources follows the set of rules given below:

The SL-RSSI of the selected CAM resource must be lower than a certain threshold $P_{\mathrm{TH}}$ set by the vehicle.

0 The CAM resource must not be selected if it is not sensed during $T_{\text {sense }}$.

O Out of all available CAM resources, already reserved resources must not be selected.

Following half-duplex transmission, a vehicle cannot sense the channel while it transmits.

If the remaining candidate CAM resources are less than $20 \%$ of the total available CAM resources, the threshold $P_{\mathrm{TH}}$ is increased by $3 \mathrm{~dB}$ and step 2 is repeated.

- $\quad$ Step 3. CAM resource selection: After identifying the best $20 \%$ of CAM resources from $L_{C A M-R}$, the vehicle randomly selects transmission CAM resources from among them. The vehicle can reserve the same CAM resources for a random number of subsequent transmissions with the same transmission interval $T_{\mathrm{CAM}}$. The minimum and maximum number of subsequent transmissions $\left(n_{\min }\right.$ and $n_{\max }$ ) before reallocation are defined by the MAC layer [17]. The 3GPP mandated values for $n_{\min }$ and $n_{\max }$ are depicted in Table 1.

- $\quad$ Step 4. CAM resources reselection: After transmitting each packet, the SB-SPS counter decreases, indicating the number of remaining consecutive packets. If the vehicle encounters zero SB-SPS counters, it can either keep the resources with a probability $p_{\mathrm{k}}$ or reselect CAM resources with a probability of $1-p_{\mathrm{k}}$.

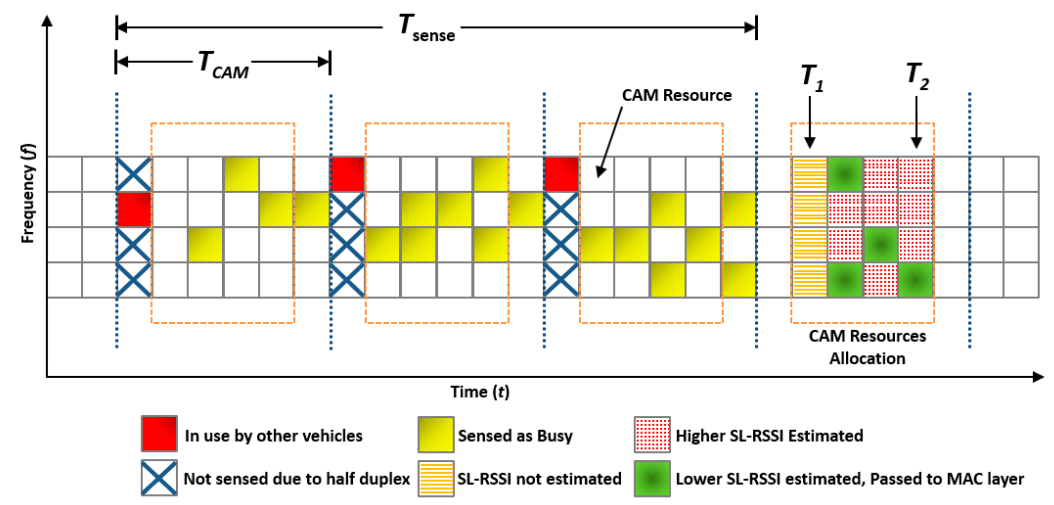

Figure 4. The procedure for sensing-based semi-persistent scheduling (SB-SPS).

Table 1. Physical and MAC layer parameters for Mode 4 along with the values defined by 3GPP.

\begin{tabular}{ccc}
\hline Parameters & Definition & 3GPP constraints \\
\hline Physical layer & Channel sensing time & \\
\hline$T_{\text {sense }}$ & Minimum threshold for the power level & 1 sec (mandated) \\
$P_{\mathrm{TH}}$ & Portion of CAM resources selected for transmission & 0.2 (mandated) \\
$R_{\mathrm{sel}}$ & First subframe for a new allocation & $\leq 4$ \\
$T_{1}$ & Last subframe for a new allocation & $\geq 20, \leq 100$ \\
$T_{2}$ & & \\
MAC layer & & 5 (mandated) \\
\hline$n_{\min }$ & Minimum number of CAM periods before reallocation & 15 (mandated) \\
$n_{\max }$ & Maximum number of CAM periods before reallocation & $\in[0,0.8]$ \\
$p_{\mathrm{k}}$ & Probability of maintaining same CAM resources &
\end{tabular}




\section{Proposed System}

This section presents the simulation environment and setup, followed by the description of the performance evaluation criteria. The proposed A-TPC algorithm is also discussed.

\subsection{Simulation Environment and Setup}

To analyze the performance of 3GPP C-V2X Mode 4 performance, this section presents the simulation setup and key assumptions taken into account. An LTEV2Vsim simulator [33], specifically designed to evaluate the performance of resource allocation algorithms in C-V2X, is employed to conduct the simulations. The block diagram for the simulator is shown in Figure 5 . It is assumed that all vehicles have CAM of the same size, generated at the same frequency and broadcasted with the same MCS settings within each traffic scenario. With these assumptions, we can ensure that the CAM resources for all vehicles are identical. The largest CAM size defined by 3GPP (300 bytes) is considered. The CAM transmission frequency is taken to be $10 \mathrm{~Hz}$. As each vehicle in C-V2X Mode 4 broadcasts the CAM, all other vehicles are considered to be potential receivers. As the distance between the vehicle transmitting the CAM and the vehicle receiving it increases, the importance of CAM decreases. Additionally, the increasing distance results in the hidden-node problem; therefore, the awareness distance is set to $100 \mathrm{~m}$ in urban scenarios and $200 \mathrm{~m}$ for the highway scenario. All vehicles within this distance range from the CAM transmitting vehicle are considered its neighbours. The major steps involved during the simulation are as follows:

- $\quad$ Step 1. System initialization: In the first step, the initialization procedure with the parameters defined in the configuration files is executed. The number of available CAM resources in each CAM period is evaluated. All the parameters have pre-defined values for each traffic scenario in the configuration files to facilitate the initialization process.

- $\quad$ Step 2. First CAM resources allocation: To reduce the initialization overhead, the first CAM resource allocation is performed for all the vehicles in each traffic scenario with the initialized parameters to mimic autonomous scheduling and perfect position estimation. Using this approach, collisions in resource allocation due to the appearance of many vehicles in a traffic scenario at a specific time interval can be avoided.

- Step 3. Simulation Cycle: After system initialization, the main part of the simulation, termed the simulation cycle, is executed and this is performed repeatedly for a defined simulation length $\mathrm{T}_{\mathrm{sim}}$. The process consists of updating the position of the vehicle, followed by the core step of quality assessment by calculating the received signal-to-interference-plus-noise ratio (SINR). The error estimation can be performed by determining the success or failure of CAM transmission using a defined minimum received SINR level for each traffic scenario. For a CAM transmission from vehicle $\mathrm{x}$ to vehicle $\mathrm{y}$, the received SINR formulation from [33] can be given as:

$$
\operatorname{SINR}_{x y}=\frac{\left(P_{\mathrm{TX} \_\mathrm{RB}} \cdot G_{\mathrm{rx}}\right) /\left(P L \cdot D_{x y}{ }^{\beta}\right)}{\left(K_{\mathrm{si}} \cdot P_{\mathrm{TX} \_\mathrm{RB}}+P_{\mathrm{n}}\right)+\sum_{\substack{k \in K_{\mathrm{n}} \\ k \neq y}}\left(P_{\mathrm{TX} \_\mathrm{RB}} \cdot G_{\mathrm{rX}}\right) /\left(P L \cdot D_{k y}{ }^{\beta}\right)}
$$

where $P_{\text {TX_RB }}$ is the uplink transmit power per resource block, $G_{r X}$ is the receiver antenna gain, $\mathrm{PL}$ is the path loss at $1 \mathrm{~m}, \mathrm{D}_{\mathrm{xy}}$ is the distance between the transmitter and receiver, $\beta$ represents the loss exponent, $P_{n}$ is the noise power level for each resource block, and $K_{n}$ represents the set of vehicles using CAM resources for the transmission. $K_{s i} \in[0,1]$ is the self-cancellation parameter. As the half-duplex mode is assumed in this work, $\mathrm{K}_{\mathrm{si}}=0$ is utilized. After evaluating the packet errors, CAM resources are reassigned for those vehicles which observed collisions within this awareness range. The task of CAM resources reallocation is performed by each vehicle independently, depending on the MAC layer 
parameter settings. At this step, the importance of the resource allocation algorithm becomes vital, as an efficient reallocation can improve the system performance.

- $\quad$ Step 4. Performance evaluation: This is the last step performed after the simulation cycle is completed, when the output performance metrics for each traffic scenario is evaluated. In this case, we focus on the packet reception ratio (PRR) for the CAM transmission defined as:

$$
\text { PRR }=\frac{\text { No. of neighbours correctly decoding CAM }}{\text { Total number of neighbours }}
$$

Following this step, the output files are generated and saved for further evaluation.

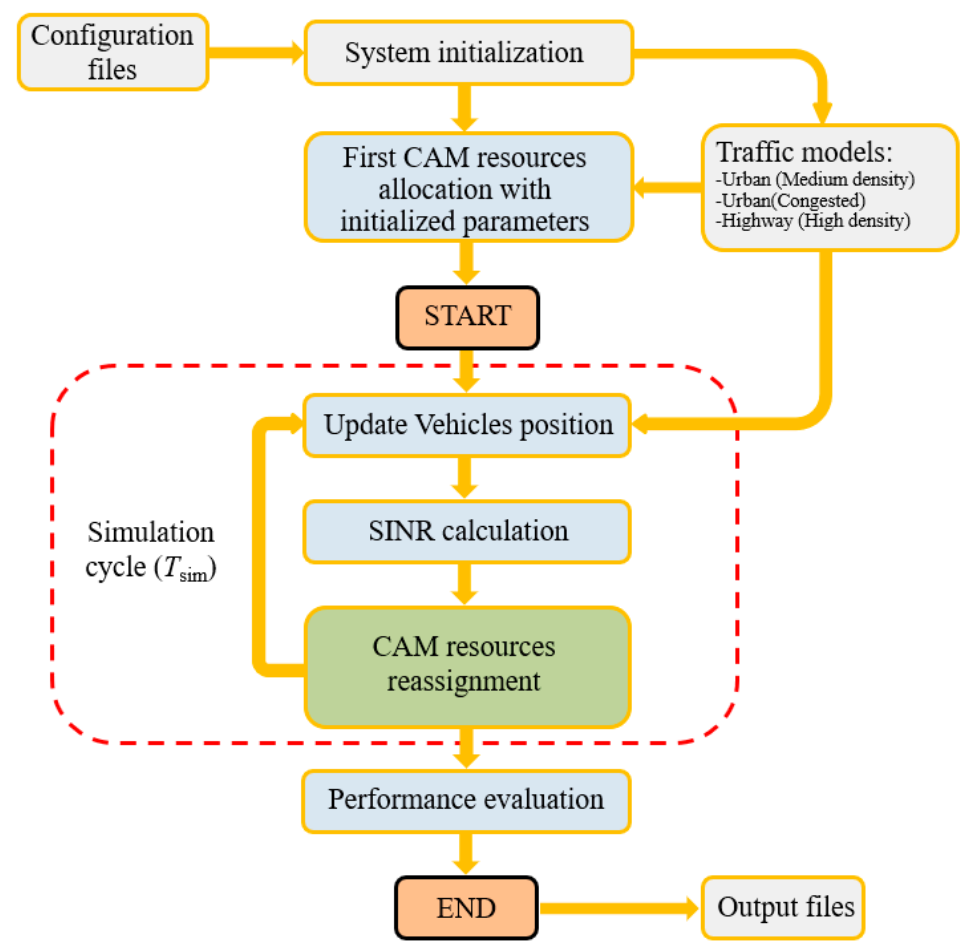

Figure 5. LTEV2Vsim simulator.

To perform a fair comparison with the power adaptation algorithm presented in [30], a 4-km section of a highway with six lanes (three for each direction) was adopted by assuming a fixed vehicle density. The parameters used for this part of the simulation, similar to those adopted in [30], are summarized in Table 2. Afterward, to evaluate the resource allocation algorithm, we employed three traffic scenarios. For the first scenario, an urban traffic trace with a size of $1.85 \times 1.85 \mathrm{~km}^{2}$ and a medium density of vehicles is considered. The number of vehicles is averaged to 925 , with 14.8 neighbours. The awareness range is considered to be $100 \mathrm{~m}$. This scenario represents sparse traffic density for the analysis. In the second case, a $1.6 \times 1.3 \mathrm{~km}^{2}$ urban traffic trace with 667 vehicles is considered. Each vehicle in this scenario has 25.4 neighbours, representing a congested scenario. A similar awareness range of $100 \mathrm{~m}$ is considered. This is followed by a high-density highway scenario, with approximately $16 \mathrm{~km}$ of a six-lane highway (three for each direction) considered. The number of vehicles is 2015 in this scenario, averaging 49.4 neighbours in the awareness range of $200 \mathrm{~m}$ around each vehicle. Following the 3GPP guidelines, a WINNER+ model scenario B1 is considered. The density of the vehicles is specified by the parameter $\rho$ in the simulation. The threshold value for the vehicle density $\rho_{\mathrm{TH}}$ is set for each scenario by adding the standard deviation in the average number of vehicles in each scenario (standard deviation of 8.8, 25.4, and 12.5 is considered for each scenario, respectively). Each vehicle can determine the density of vehicles within the awareness range by monitoring the received sidelink RSSI 
measurements. An efficient monitoring of the sidelink RSSI measurement plays a vital role during the setup of propagation models for $\mathrm{V} 2 \mathrm{~V}$ communications [34]. From the perspective of performance for the CAM reception, a packet is considered to be received successfully and decoded correctly if the received SINR is above a certain pre-defined minimum threshold. The simulation parameters for each traffic scenario are summarized in Table 3.

\subsection{Adaptive Transmit Power Control Algorithm}

In this section, the proposed A-TPC algorithm is explained. The algorithm is executed during the CAM resources reallocation step in the simulation to set the transmit power of each vehicle. The steps involved in the proposed algorithm are illustrated in Figure 6. The function of each step to determine the transmit power for CAM transmission using SB-SPS is as described below:

- $\quad$ Step 1. Compute SL-RSSI matrix: In the first step, the vehicles performing reallocation of the CAM resources are listed according to their vehicle ID in the ID list $\left(\mathrm{L}_{\mathrm{ID}}\right)$. To determine the number of vehicles performing reallocation, the index of $\mathrm{L}_{\mathrm{ID}}$ is computed. Afterward, the SL_RSSI matrix for each vehicle in LID is computed by summing the SL-RSSI values in each subframe.

- $\quad$ Step 2: Interference calculation: In this step, the interference experienced by each vehicle over the sidelink is calculated by summing the SL-RSSI values from each neighbouring vehicle in the same subframe with the CAM resources. This interference is termed as ISL_RSSI.

- $\quad$ Step 3. Transmit power allocation: In this step, the transmit power is allocated to each vehicle during the process of CAM resources reallocation based on the interference and the density of vehicles in each scenario. The density of vehicles $=\rho$ is defined as the number of vehicles per $\mathrm{km}$ in each scenario. The maximum vehicle density $=\rho_{\mathrm{TH}}$ for each scenario is defined in the simulation. For a vehicle density lower then $=\rho_{\mathrm{TH}}$, our proposed algorithm allocates the maximum transmit power of $23 \mathrm{dBm}$ for CAM transmission. However, with higher vehicle density, the chances of failed CAM transmission increases due to a significant increase in interference. Following the ETSI ITS recommendation [18], the transmit power is initialized by adopting the 3GPP Mode 4 at $10 \mathrm{dBm}$, and adaptive power control is performed based on the amount of interference in the system. Depending on the MCS adopted in each scenario, the interference threshold $\mathrm{I}_{\mathrm{TH}}$ can be obtained by using Equation (1) with the minimum required SINR for each MCS defined in Table 3. Interference threshold $\mathrm{I}_{\mathrm{TH}}$ is defined as the maximum allowable interference level for achieving the minimum required SINR. By comparing the $\mathrm{I}_{\mathrm{SL} \_ \text {RSSI }}$ to an interference threshold $\mathrm{I}_{\mathrm{TH}}$, the proposed algorithm increases the initialized transmit power by $\Delta_{\mathrm{P}}$ at every CAM resource reallocation, up to the maximum transmit power limit $P_{\max }=23 \mathrm{dBm}$.

The pseudo-code for the proposed A-TPC Algorithm 1 is given below: 


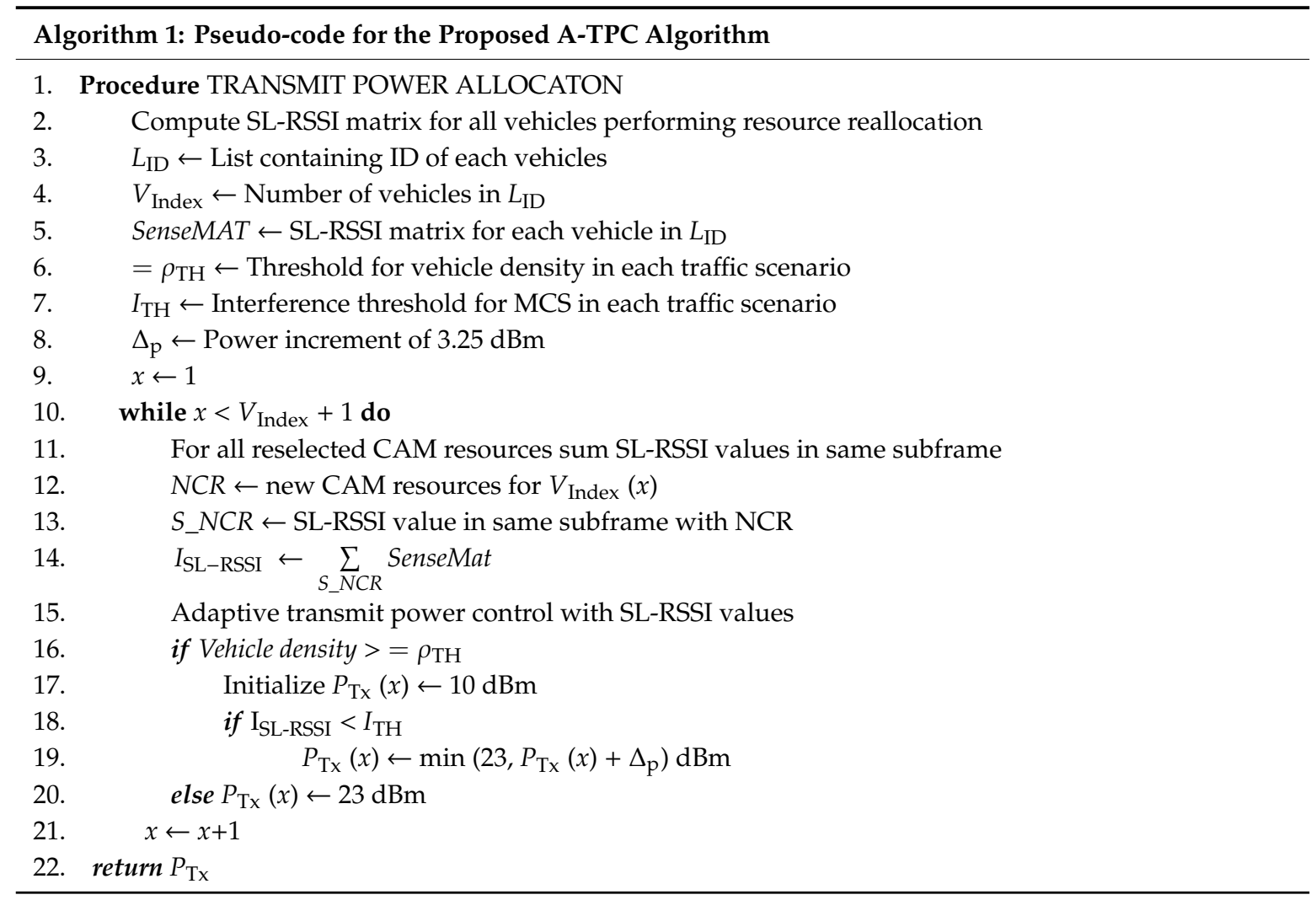

Table 2. Summary of parameters and settings for Simulation 1 [30].

\begin{tabular}{cc}
\hline Parameters & Values \\
\hline \multicolumn{2}{c}{ Simulation inputs } \\
\hline Traffic scenario & 4-km highway $($ three lanes per direction) \\
Vehicle density $(=\rho)$ & 100,200 \\
Vehicles speed & $114 \mathrm{~km} / \mathrm{h}$ \\
Awareness range & $150 \mathrm{~m}$ \\
CAM periodicity $\left(f_{\mathrm{CAM}}\right)$ & $10 \mathrm{~Hz}$ \\
CAM size $\left(B_{\text {CAM }}\right)$ & $300 \mathrm{bytes}$ \\
Propagation model & WINNER+ \\
Shadowing variance & LOS $3 \mathrm{~dB}, \mathrm{NLOS} 4 \mathrm{~dB}$ \\
MCS & 4 \\
Minimum SINR & $2.76 \mathrm{~dB}$ \\
\hline Physical and MAC layer settings \\
\hline$T_{\text {sense }}$ & $1 \mathrm{~s}$ \\
$P_{\mathrm{TH}}$ & $-110 \mathrm{dBm}$ \\
$R_{\text {sel }}$ & 0.2 \\
$T_{1}$ & 1 \\
$T_{2}$ & 100 \\
$n_{\min }$ & 5 \\
$n_{\max }$ & 15 \\
$p_{\mathrm{k}}$ & 0 \\
\hline
\end{tabular}

The proposed algorithm adjusts the uplink transmit power for the transmission of CAM resources by assigning a higher transmit power to vehicles in the event of lower traffic density and a lower value in the event of congestion. The algorithms adaptively increase the transmit power in the event of congestion through evaluation interference at every CAM resource reallocation. 
Table 3. Summary of parameters and settings for Simulation 2.

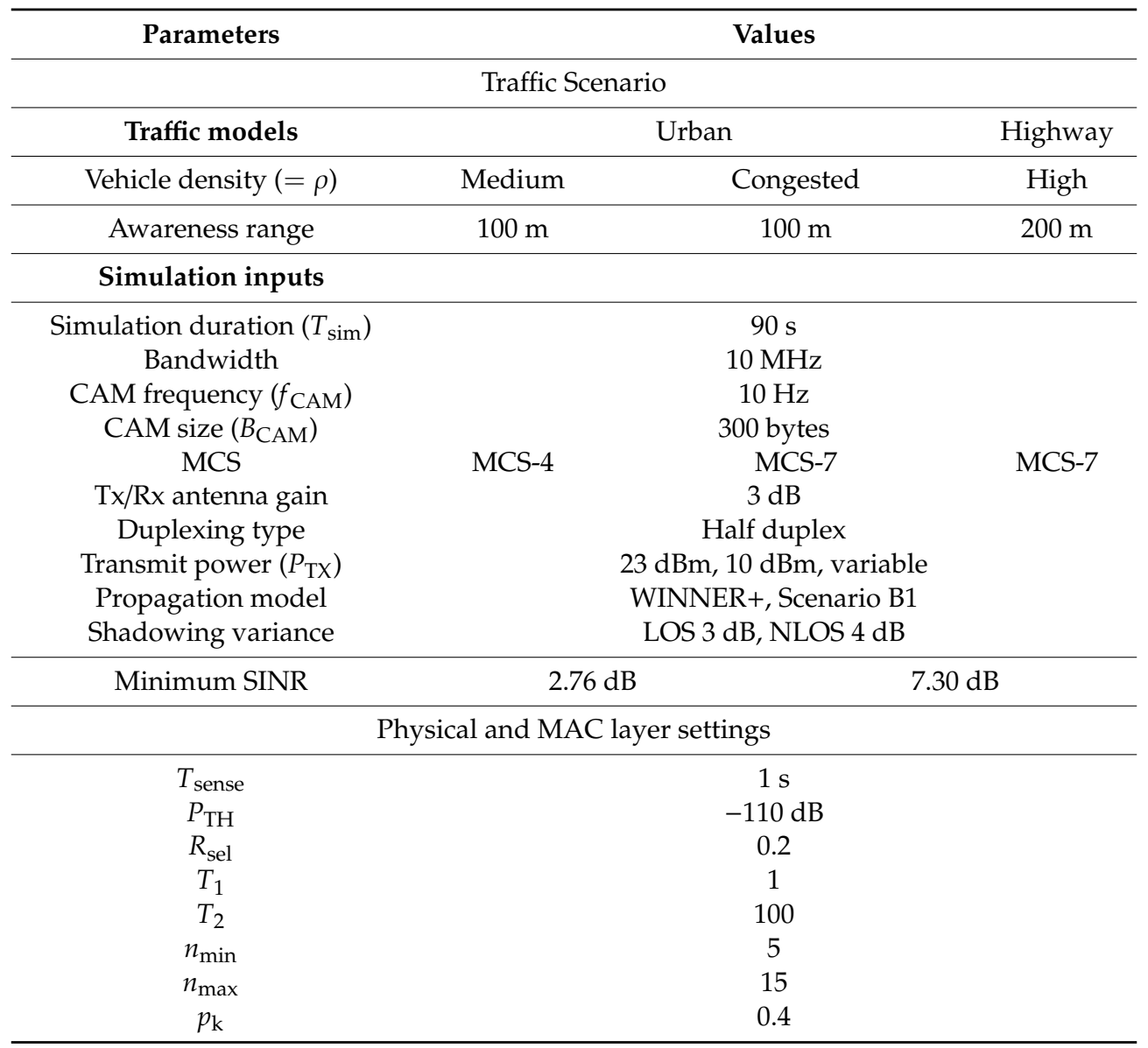

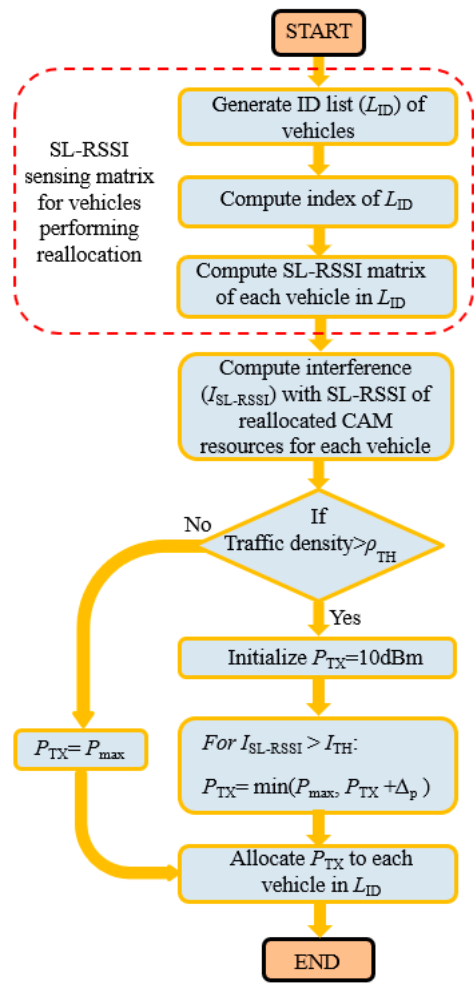

Figure 6. Flow graph for the proposed adaptive power control algorithm. 


\section{Simulation Results}

This section presents the simulation results for the proposed A-TPC algorithm for C-V2X Mode 4. The simulations are performed by adopting three realistic traffic scenarios and the evaluation of the performance of the proposed algorithm is quantified in terms of the PRR described in Section 3.1. To evaluate the impact of the transmit power on the system performance, the proposed algorithm is compared to the power adaptation algorithm presented in [30] for a highway scenario with a fixed vehicle density. Afterward, the performance evaluation for the three TPC schemes is presented for the three realistic traffic scenarios, starting with the $3 \mathrm{GPP}$ standard Mode 4 at $P_{\mathrm{TX}}=23 \mathrm{dBm}$, followed by ETSI ITS recommendation for Mode 4 at $P_{\mathrm{TX}}=10 \mathrm{dBm}$, and finally the proposed A-TPC algorithm.

\subsection{Performance Comparison with Existing Scheme}

The simulation is first performed to show the comparison of the proposed algorithm by adopting the same parameter settings as the power adaptation algorithm presented in [30]. The algorithm proposed in [30] considers the power adaptation for the vehicles in a highway scenario only with a fixed traffic density. For lower traffic density $(=\rho=100 \mathrm{veh} / \mathrm{km}), 23 \mathrm{dBm}$ transmit power is assigned to all the vehicles, whereas for a high traffic density $(=\rho=200 \mathrm{veh} / \mathrm{km}), 10 \mathrm{dBm}$ is assigned to all the vehicles in the network. Based on the interference level, the vehicles can adopt the $23 \mathrm{dBm}$ transmit power level for the latter case with high traffic density. However, this approach of adopting a fixed vehicle density is not practical, as with this assumption, all vehicles tend to perform the power adaptation, resulting in a decrease for available CAM resources during the sensing time for each vehicle. Also, switching from $10 \mathrm{dBm}$ to $23 \mathrm{dBm}$ with a step of $13 \mathrm{dBm}$ can significantly increase the interference among vehicles, resulting in severe performance degradation. The details of the algorithm can be found in [30]. The traffic model adopted for this part of the simulation is a highway scenario with a fixed vehicle density. The simulation settings and parameter values used in the comparison are presented in Table 2. The results of the comparison are depicted in Figure 7. For the first part, a lower vehicle density is considered, that is, $=\rho=100 \mathrm{veh} / \mathrm{km}$. The scenario is sparse, signifying low interference and thus resulting in lower SL-RSSI values. Both algorithms adopt a maximum transmit power of $23 \mathrm{dBm}$ following the 3GPP recommendation. It should be noted that for the distance range beyond $100 \mathrm{~m}$, the lower transmit power of $10 \mathrm{dBm}$ cannot ensure sufficient minimum SINR requirements at the receiving vehicle, resulting in degradation of the PRR.

For a higher traffic density of $=\rho=200 \mathrm{veh} / \mathrm{km}$, the power adaptation algorithm in [30] assigns a lower transmit power of $10 \mathrm{dBm}$ to vehicles experiencing high interference and $23 \mathrm{dBm}$ to vehicles experiencing lower interference. However, in the event of high vehicle density, our proposed algorithm assigns $10 \mathrm{dBm}$ to all vehicles performing reallocation, resulting in an overall decrease in interference. As the vehicles perform the next reallocation, they increase their transmit power gradually instead of a sudden jump to $23 \mathrm{dBm}$, thus maintaining a reasonable interference level in the network. This impact can be seen clearly in Figure 7, where our proposed algorithm outperforms the conventional TPC schemes by adopting a lower transmit power of $10 \mathrm{dBm}$ up to a distance range of $130 \mathrm{~m}$. As the distance between the source and destination increases, the transmit power is gradually increased to ensure acceptable received SINR level at the receiving vehicle. Comparing both curves in Figure 7 for the two adopted vehicle density values, it can be seen clearly that in a dense vehicular network, the role of power control becomes vital to performance improvement. However, the limitation in this case is the use of fixed traffic density values, which is not the case in a real environment. Therefore, in the following section, we further investigate the proposed algorithm by adopting realistic traffic scenarios where the traffic density is not assumed to be fixed around each vehicle. 


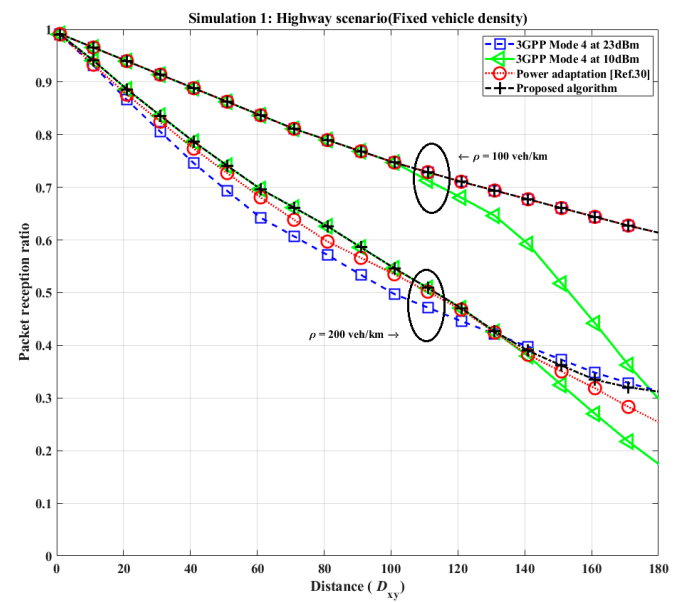

Figure 7. Packet reception ratio as a function of the distance between the transmitter and receiver $\left(D_{\mathrm{xy}}\right)$ in highway scenario with fixed traffic density.

\subsection{Performance Evaluation of Proposed Algorithm}

This section presents the performance evaluation of the proposed algorithm for the three traffic scenarios using the standard 3GPP Mode 4 physical and MAC layer parameters defined in Table 1 and the simulation parameters summarized in Table 3. The first goal of this simulation setup is to find the appropriate MCS level $(4,7$, or 14) for each traffic scenario instead of the conventional approach of employing the same MCS level in every scenario. The choice of MCS determines the number of subchannels occupied by the CAM; 1-CAM occupies four subchannels with MCS-4, 2-CAMs occupy two subchannels with MCS-7, and 4-CAMs occupy four subchannels with MCS-14. Therefore, it is vital to adopt the appropriate MCS level for each traffic scenario. Figure 8 shows the impact of various MCS levels on the PRR for each traffic scenario. The higher PRR for the highway scenario demonstrates that the signal propagation in this scenario is dominated by line-of-sight (LOS) communication. The PRR results indicate that there exists a trade-off between the available CAM resources versus the required minimum received SINR level defined for each MCS in Table 3. A higher MCS ensures more CAM resources for transmission, while the minimum received SINR level required to determine the success of CAM transmission also becomes higher. This results in frequent failures in CAM transmission due to insufficient received power at the receiver and unnecessary resource reallocations, introducing latency in C-V2X communication. Taking this into consideration to analyse the results shown in Figure 8, it is appropriate to choose MCS-4 for an urban scenario with medium traffic density while choosing MCS-7 for the two traffic scenarios with a high density of vehicles.

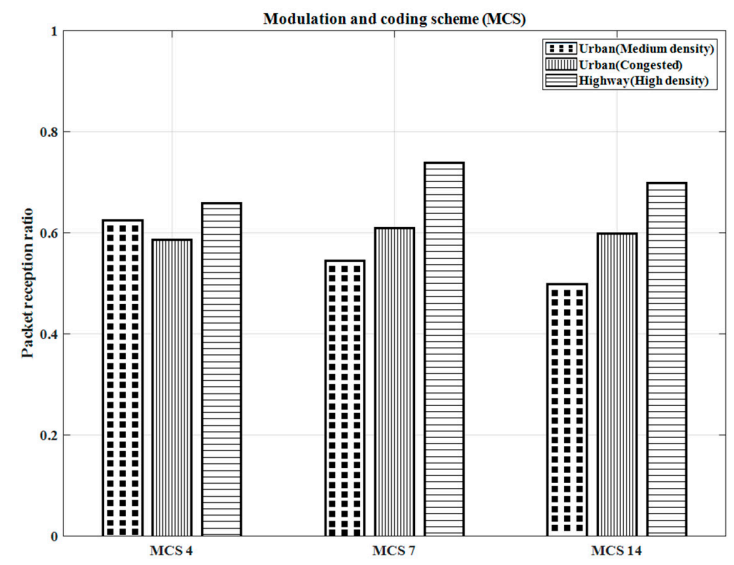

Figure 8. Impact of variations in modulation and coding schemes (MCS) on the packet reception ratio. 
To demonstrate the impact of the proposed algorithm, the system performance in terms of the PRR for the three deployment scenarios is presented in this section. The urban scenario with a medium traffic density is first considered. In Figure 9, it can be seen clearly that up to $120 \mathrm{~m}$, the conventional $23 \mathrm{dBm}$ and $10 \mathrm{dBm}$ choices for the transmit power show similar trends in terms of PRR. Beyond this distance, the lower transmit power results in degradation of PRR due to insufficient received power at the receiver. As the traffic density for this scenario is medium, our proposed algorithm opts for the higher transmit power level of $23 \mathrm{dBm}$, ensuring similar performance to the standard 3GPP Mode 4 algorithm.

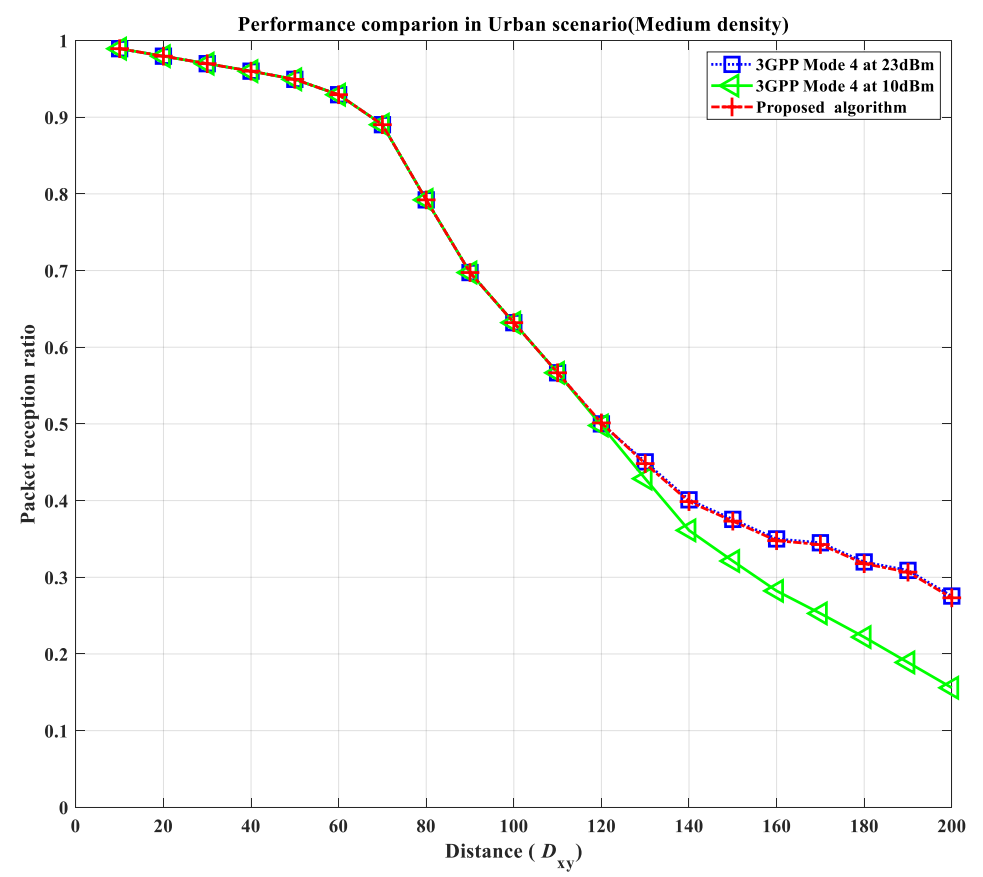

Figure 9. Packet reception ratio as a function of the distance between transmitter and receiver $\left(D_{\mathrm{xy}}\right)$ in an urban scenario with medium traffic density.

As mentioned earlier, the role of TPC schemes becomes dominant for traffic scenarios with higher vehicle density. Following this observation, the impact of the A-TPC mechanism can be well observed in the urban scenario with congested traffic, where the congestion results in an increase of vehicle density, making the role of the power control algorithm dominant. For a distance range of 31 to $120 \mathrm{~m}$ shown in Figure 10, the impact of congestion and the consequent generation of interference becomes dominant. A higher transmit power results in a significant increase in the interference, while transmitting at lower power ensures higher PRR by keeping the interference level in an acceptable range. The results for the proposed algorithm demonstrate that in congested environments, efficient evaluation of the interference and accordingly adapting the transmit power can result in significant performance gain. The proposed algorithm tends to opt for a lower transmit power value of $10 \mathrm{dBm}$ up to $120 \mathrm{~m}$ and gradually increases the transmit power to ensure satisfactory received power at the receiver. The results confirm that the proposed algorithm outperforms the conventional TPC schemes. A similar trend can be seen in Figure 11 for the highway scenario with high vehicle density, where the proposed algorithm outperforms the conventional schemes, particularly in a distance range of 10 to $230 \mathrm{~m}$, while maintaining a PRR close to the 3GPP Mode 4 algorithm beyond this distance. The importance of performance improvement in the lower distance range is vital for CAM transmissions as they are intended to carry the safety-related information for C-V2X with a significant improvement at shorter distances. It is important to notice from the comparison of Figures 7 and 11, the impact of adopting the correct MCS level, as well as the consideration of realistic traffic scenarios rather than using a fixed vehicle density. For a distance range of $10 \mathrm{~m}$ to $200 \mathrm{~m}$, the results show significant gain in terms of PRR. 
Additionally, it is worth mentioning that in realistic traffic scenarios, the proposed algorithm tends to keep the transmit power lower compared to a traffic scenario with a fixed user density, thus supporting our intention to adopt the TPC mechanism. A similar trend can be observed by comparing Figure 9 to Figures 10 and 11, where the advantage of using a higher MCS level pays off in terms of significant performance gain in the PRR.

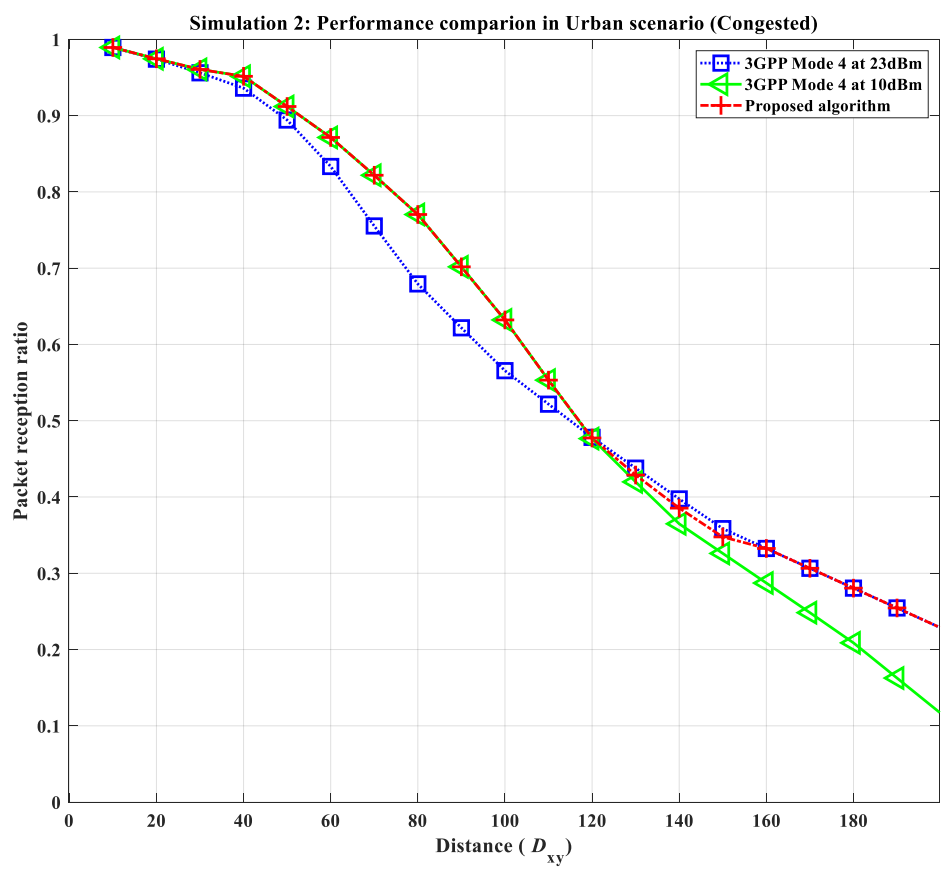

Figure 10. Packet reception ratio as a function of the distance between the transmitter and receiver $\left(D_{\mathrm{xy}}\right)$ in an urban scenario with congested traffic.

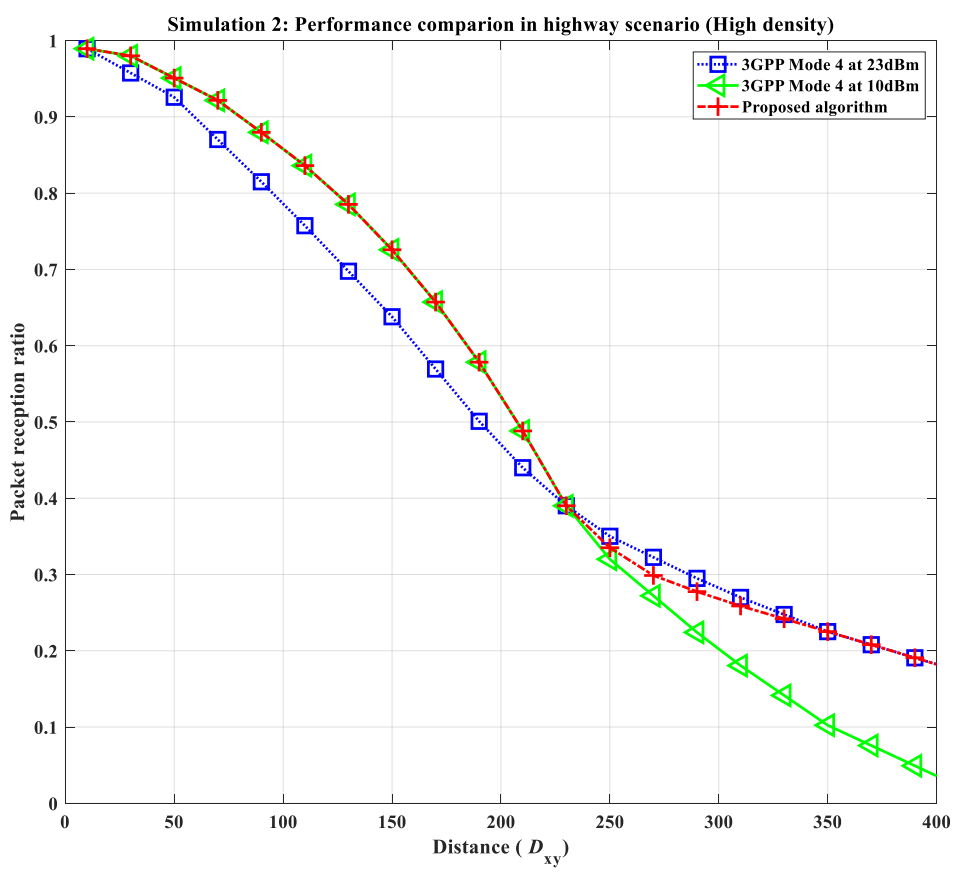

Figure 11. Packet reception ratio as a function of the distance between the transmitter and receiver $\left(D_{\mathrm{xy}}\right)$ in a highway scenario with high traffic density.

Security is an important aspect in V2X communication, because V2X messages convey sensitive and life critical real-time information that needs to be secured against cyber-attacks [35]. Security protocols 
must be implemented with low communication overhead due to time constraint and low computation complexity to exchange quick and safe information. Our future work will be focused on the design and implementation of enhanced security algorithms for C-V2X communications.

\section{Conclusions}

In this work, the impact of TPC on the performance of the C-V2X Mode 4 algorithm is investigated. An A-TPC algorithm based on SB-SPS is proposed to implement the power control mechanism for CAM transmission. The proposed algorithm operates during the process of CAM resources reallocation. The proposed algorithm is investigated for three realistic traffic scenarios. The results demonstrate that for traffic scenarios with high vehicle density, the proposed algorithm outperforms the conventional TPC schemes. While the improvement seems to be negligible in traffic scenarios with lower vehicle density, it becomes significant in the event of congestion. Future research will be focused on investigating coexistence with $802.11 \mathrm{p}$ along with the design and implementation of enhanced security algorithms for C-V2X communications.

Author Contributions: A.H. and S.-H.H. contributed to the main idea of this research. A.H. performed the simulations. A.H. and S.-H.H. contributed to the writing of this article. This research activity was planned and executed under the supervision of S.-H.H.

Funding: This research received no external funding.

Conflicts of Interest: The authors declare no conflicts of interest regarding the publication of this article.

\section{References}

1. Chen, S.; Hu, J.; Shi, Y.; Peng, Y.; Fang, J.; Zhao, R.; Zhao, L. Vehicle-to-Everything (v2x) Services Supported by LTE-Based Systems and 5G. IEEE Commun. Stand. Mag. 2017, 1, 70-76. [CrossRef]

2. Wang, M.; Winbjork, M.; Zhang, Z.; Blasco, R.; Do, H.; Sorrentino, S.; Belleschi, M.; Zang, Y. Comparison of LTE and DSRC-Based Connectivity for Intelligent Transportation Systems. In Proceedings of the IEEE Vehicular Technology Conference, Sydney, NSW, Australia, 4-7 June 2017.

3. Fakhfakh, E.; Hamouda, S.; Tabbane, S. Enhanced traffic offloading with D2D communications under noise rise constraint. In Proceedings of the IEEE Symposium on Computers and Communication, Messina, Italy, 27-30 June 2016; pp. 1112-1116.

4. Tang, H.; Ding, Z. Mixed Mode Transmission and Resource Allocation for D2D Communication. IEEE Trans. Wirel. Commun. 2016, 15, 162-175. [CrossRef]

5. Kim, J.; Kim, S.; Bang, J.; Hong, D. Adaptive Mode Selection in D2D Communications Considering the Bursty Traffic Model. IEEE Commun. Lett. 2016, 20, 712-715. [CrossRef]

6. Shrestha, R.; Bajracharya, R.; Nam, S.Y. Challenges of Future VANET and Cloud-Based Approaches. Wirel. Commun. Mob. Comput. 2018, 2018, 1-15. [CrossRef]

7. Morgan, Y.L. Notes on DSRC \& WAVE standards suite: Its architecture, design, and characteristics. IEEE Commun. Surv. Tutorials 2010, 12, 504-518.

8. Zeadally, S.; Hunt, R.; Chen, Y.S.; Irwin, A.; Hassan, A. Vehicular ad hoc networks (VANETS): Status, results, and challenges. Telecommun. Syst. 2012, 50, 217-241. [CrossRef]

9. Caballero-Gil, P.; Caballero-Gil, C.; Molina-Gil, J. How to build vehicular ad-hoc networks on smartphones. J. Syst. Archit. 2013, 59, 996-1004. [CrossRef]

10. Caballero-Gil, C.; Caballero-Gil, P.; Molina-Gil, J. Merging sub-networks in VANETs by using the IEEE 802.11xx protocols. Peer Peer Netw. Appl. 2015, 8, 664-673. [CrossRef]

11. Seppälä, J.; Koskela, T.; Chen, T.; Hakola, S. Network controlled Device-to-Device (D2D) and cluster multicast concept for LTE and LTE-A networks. In Proceedings of the IEEE Wireless Communications and Networking Conference (WCNC), Cancun, Quintana Roo, Mexico, 28-31 March 2011; pp. 986-991.

12. Caballero-Gil, C.; Caballero-Gil, P.; Molina-Gil, J. Self-Organized Clustering Architecture for Vehicular Ad Hoc Networks. Int. J. Distrib. Sens. Networks 2015, 2015, 384869. [CrossRef]

13. Abbas, F.; Fan, P.; Khan, Z. A Novel Low-Latency V2V Resource Allocation Scheme Based on Cellular V2X Communications. IEEE Trans. Intell. Transp. Syst. 2018, 2185-2197. [CrossRef] 
14. Qi, Y.; Mach, T. QoS and Coverage Aware Dynamic High Density Vehicle Platooning (HDVP). In Proceedings of the 2018 IEEE 88th Vehicular Technology Conference, Chicago, IL, USA, 27-30 August 2019; pp. 1-5.

15. Tian, D.; Li, W.; Wu, G.; Boriboonsomsin, K.; Barth, M.; Rajab, S.; Bai, S. Evaluating the effectiveness of v2v-based lane speed monitoring application: A simulation study. In Proceedings of the IEEE Conference Intelligent Transportation System Proceedings, ITSC 2016, Rio de Janeiro, Brazil, 8-29 August 2016; pp. 1592-1597.

16. Specification, T. TS 136 213-V8.8.0-LTE; Evolved Universal Terrestrial Radio Access (E-UTRA); Physical Layer Procedures (3GPP TS 36.213 Version 8.8.0 Release 8); European Telecommunications Standards Institute: Sophia-Antipolis, France, 2009.

17. 3GPP. TS 136 321-V8.2.0-LTE; Evolved Universal Terrestrial Radio Access (E-UTRA); Medium Access Control (MAC) protocol specification (3GPP TS 36.321 version 8.2.0 Release 8). 2008. Available online: https://portal. 3gpp.org/desktopmodules/Specifications/SpecificationDetails.aspx?specificationId=2437 (accessed on 25 May 2019).

18. Communications, V.; Service, A.B. ETSI EN 302 637-2-V1.3.2-Intelligent Transport Systems (ITS)-Vehicular Communications-Basic Set of Applications-Part 2: Specification of Cooperative Awareness Basic Service; European Telecommunications Standards Institute: Sophia-Antipolis, France, 2010; Volume 1, pp. 1-22.

19. Boban, M.; Kousaridas, A.; Manolakis, K.; Eichinger, J.; Xu, W. Connected Roads of the Future: Use Cases, Requirements, and Design Considerations for Vehicle-to-Everything Communications. IEEE Veh. Technol. Mag. 2018, 13, 110-123. [CrossRef]

20. Haider, A.; Sinha, R.S.; Hwang, S.H. Investigation of Open-Loop Transmit Power Control Parameters for Homogeneous and Heterogeneous Small-Cell Uplinks. ETRI J. 2018, 40, 51-60. [CrossRef]

21. Molina-Masegosa, R.; Gozalvez, J. System Level Evaluation of LTE-V2V Mode 4 Communications and Its Distributed Scheduling. In Proceedings of the 2017 IEEE 85th Vehicular Technology Conference, Sydney, NSW, Australia, 4-7 June 2017.

22. Wei, Y.; Chen, J.; Hwang, S.H. Adjacent vehicle number-triggered adaptive transmission for v2v communications. Sensors (Switzerland) 2018, 18, 755.

23. Bazzi, A.; Masini, B.M.; Zanella, A.; Thibault, I. On the performance of IEEE 802.11p and LTE-V2V for the cooperative awareness of connected vehicles. IEEE Trans. Veh. Technol. 2017, 66, 10419-10432. [CrossRef]

24. Yu, C.; Si, S.; Guo, H.; Zhao, H. Modeling and Performance of the IEEE 802.11p Broadcasting for Intra-Platoon Communication. Sensors 2018, 18, 2971. [CrossRef]

25. Molina-Masegosa, R.; Gozalvez, J. LTE-V for Sidelink 5G V2X Vehicular Communications. IEEE Veh. Technol. Mag. 2017, 12, 30-39. [CrossRef]

26. Jeon, Y.; Kuk, S.; Kim, H. Reducing message collisions in sensing-based semi-persistent scheduling (SPS) by using reselection lookaheads in cellular V2X. Sensors (Switzerland) 2018, 18, 4388. [CrossRef]

27. Gonzalez-Martin, M.; Sepulcre, M.; Molina-Masegosa, R.; Gozalvez, J. Analytical Models of the Performance of C-V2X Mode 4 Vehicular Communications. IEEE Trans. Veh. Technol. 2019, 68, 1155-1166. [CrossRef]

28. Nabil, A.; Marojevic, V.; Kaur, K.; Dietrich, C. Performance Analysis of Sensing-Based Semi-Persistent Scheduling in C-V2X Networks. In Proceedings of the 2018 IEEE 88th Vehicular Technology Conference (VTC-Fall), Chicago, IL, USA, 27-30 August 2018. 18601367. [CrossRef]

29. Bazzi, A.; Cecchini, G.; Zanella, A.; Masini, B.M. Study of the Impact of PHY and MAC Parameters in 3GPP C-V2V Mode 4. IEEE Access 2018, 6, 71685-71698. [CrossRef]

30. Kang, B.; Jung, S.; Bahk, S. Sensing-Based Power Adaptation for Cellular V2X Mode 4. In Proceedings of the IEEE International Symposium on Dynamic Spectrum Access Networks, DySPAN 2018, Seoul, Korea, $22-25$ October 2018; pp. 1-4.

31. Uppoor, S.; Trullols-Cruces, O.; Fiore, M.; Barcelo-Ordinas, J.M. Generation and analysis of a large-scale urban vehicular mobility dataset. IEEE Trans. Mob. Comput. 2014, 13, 1061-1075. [CrossRef]

32. Bazzi, A.; Masini, B.M.; Zanella, A.; Calisti, A. Visible light communications as a complementary technology for the internet of vehicles. Comput. Commun. 2016, 93, 39-51. [CrossRef]

33. Cecchini, G.; Bazzi, A.; Masini, B.M.; Zanella, A. LTEV2Vsim: An LTE-V2V simulator for the investigation of resource allocation for cooperative awareness. In Proceedings of the 2017 th IEEE International Conference Models Technologies for Intelligent Transportation System MT-ITS, Naples, Italy, 26-28 June 2017; pp. 80-85. 
34. Kokalj-Filipovic, S.; Greenstein, L.; Cheng, B.; Gruteser, M. Methods for extracting V2V propagation models from imperfect RSSI field data. In Proceedings of the 82nd Vehicular Technology Conference (VTC2015-Fall), Boston, MA, USA, 6-9 September 2015; pp. 1-5.

35. Alnasser, A.; Sun, H.; Jiang, J. Cyber security challenges and solutions for V2X communications: A survey. Comput. Networks 2019, 151, 52-67. [CrossRef] 